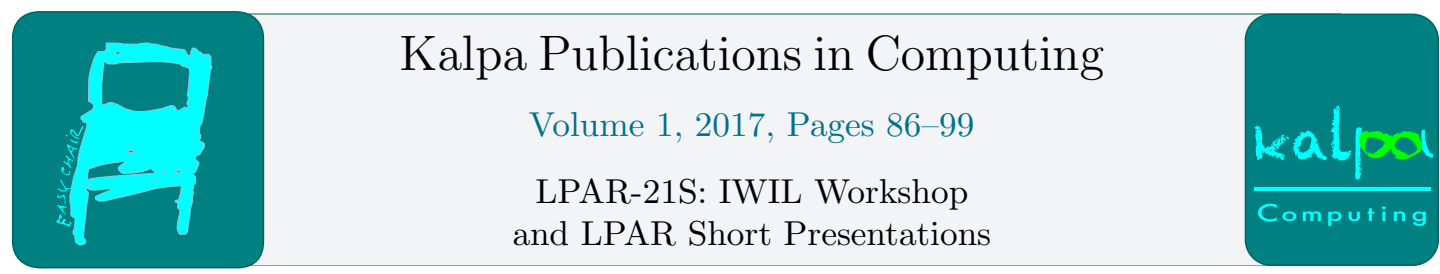

\title{
Translating C\# to Branching Symbolic Transducers
}

\author{
Olli Saarikivi ${ }^{1}$ and Margus Veanes ${ }^{2}$ \\ 1 Helsinki Institute for Information Technology HIIT \\ Aalto University \\ olli.saarikivi@aalto.fi \\ 2 Microsoft Research \\ margus@microsoft.com
}

\begin{abstract}
The paper looks at tooling aspects of transforming C\# programs into symbolic transducers with branching rules (BSTs). The latter are used for describing list comprehensions that incorporate loop-carried state. One concrete application is log analysis where input streams of data are transformed into output streams of data via intermediate pipelines of transducers. The paper presents algorithms for translating C\# to BSTs, and for exposing control state in BSTs.
\end{abstract}

\section{Introduction}

This paper discusses some of the algorithmic support underlying the tool introduced in [14] that implements effectful comprehensions and introduces the notion of symbolic transducers with branching rules (BSTs). Effectful comprehensions provide an elegant way to describe list comprehensions that incorporate loop-carried state. As a motivation, consider the problem of analyzing logs. The log on the disk is compressed, and thus the user has to first decompress the input stream of bits into bytes. Then the bytes are decoded into characters, and finally sequences of characters are deserialized or parsed into objects in a higher-level language such as C\#. Such processing from input stream of bits to output stream of bits with intermediate layers of objects is not uncommon today $[8,1,19,5]$, and applying fusion to such pipelines can be beneficial $[13,18]$. In order for such fusion techniques to be widely applicable to real world programs there must be an accessible way to specify effectful comprehensions.

While efficient fusion of transducers is important and improves efficiency akin to filter fusion [13] and deforestation [18], so is the aspect of transforming C\# programs (that are used in the frontend) into transducers (that are used in the backend). This latter aspect and the underlying tool support and algorithms used for that is the primary focus of this paper. We present a C\# interface for specifying effectful comprehensions that encapsulates state usage. The interface is similar to ones found in existing streaming libraries. We describe the algorithms that are used to translate programs that implement this interface into symbolic transducers. There are two levels of transformations. First we show how we translate C\# programs into BSTs and then how we further transform the generated BSTs to expose control states, by eliminating register dependencies, where we study partial and full register exploration algorithms for BSTs.

T.Eiter, D.Sands, G.Sutcliffe and A.Voronkov (eds.), LPAR-21S (Kalpa Publications in Computing, vol. 1), 


\section{Branching Symbolic Transducers}

We here formally define branching symbolic transducers or BSTs and give examples of how $B S T$ s capture behavior of programs. For the background logic of BSTs we assume a background structure that has an effectively enumerable background universe $\mathbb{U}$, and is equipped with a language of function and relation symbols with fixed interpretations.

We use $\tau, \iota$ and $o$ to denote types, and we write $\mathbb{U}_{\tau}$ for the corresponding sub-universe of elements of type $\tau$. The Boolean type is bool, with $\mathbb{U}_{\text {bool }}=\{$ true,false $\}$, the integer type is int, and the type of $k$-bit bit-vectors is bvk. The Cartesian product type of types $\iota$ and $o$ is $\iota \times o$. We use $\langle\cdot, \ldots, \cdot\rangle: \tau_{1} \rightarrow \cdots \rightarrow \tau_{n} \rightarrow \tau_{1} \times \cdots \times \tau_{n}$ as constructors for Cartesian product (i.e. tuple) types. For projecting the $n$th element of a Cartesian product term $x$ we use $\pi_{n}(x)$. The type $\iota^{*}$ is the type for finite sequences of elements of type $\iota$. The universe $\mathbb{U}_{\left(\iota^{*}\right)}$ is the Kleene closure $\left(\mathbb{U}_{\iota}\right)^{*}$ of the universe $\mathbb{U}_{\iota}$. We also write type $\iota^{\leq k}$ as a semantic subtype of $\iota^{*}$ of sequences of elements of length at most $k \geq 0$.

Terms and formulas are defined by induction over the background language and are assumed to be well-typed. The type $\tau$ of a term $t$ is indicated by $t: \tau$. Terms of type bool, or Boolean terms, are treated as formulas, i.e., no distinction is made between formulas and Boolean terms. All elements in $\mathbb{U}$ are also assumed to have corresponding constants in the background language and we use elements in $\mathbb{U}$ also as constants. The set of free variables in a term $t$ is denoted by $F V(t), t$ is closed when $F V(t)=\emptyset$, and closed terms $t$ have Tarski semantics $\llbracket t \rrbracket$ over the background structure. Substitution of a variable $x: \tau$ in $t$ by a term $u: \tau$ is denoted by $t[u / x]$.

A $\lambda$-term $f$ is an expression of the form $\lambda x$.t, where $x: \iota$ is a variable, and $t: o$ is a term such that $F V(t) \subseteq\{x\}$; the type of $f$ is $\iota \rightarrow o ; \llbracket f \rrbracket$ denotes the function that maps $a \in \Sigma$ to $\llbracket t[a / x \rrbracket \in \Gamma$. As a convention, $f$ and $g$ stand for $\lambda$-terms. A $\lambda$-term of type $\iota \rightarrow$ bool is called a $\iota$-predicate. We write $\varphi$ and $\psi$ for $\iota$-predicates and, for $a \in \Sigma$, we write $a \in \llbracket \varphi \rrbracket$ for $\llbracket \varphi \rrbracket(a)=$ true. We often treat $\llbracket \varphi \rrbracket$ as a subset of $\Sigma$. Given a $\lambda$-term $f=(\lambda x . t): \iota \rightarrow o$ and a term $u: \iota, f(u)$ stands for $t[u / x]$. A predicate $\varphi$ is unsatisfiable when $\llbracket \varphi \rrbracket=\emptyset$; satisfiable, otherwise.

The main building block of an BST is a rule. A rule is an expression that denotes a partial function corresponding to a straight-line conditional statement of a program that may yield outputs, produce updates and raise exceptions. We first provide an inductive definition of rules that omits type annotations. We then define additional well-formedness criteria and the semantics for rules.

- Undef is the exception rule.

- If $f$ is a $\lambda$-term then $\operatorname{Base}(f)$ is a basic rule.

- If $\varphi$ is a predicate and $r_{1}, r_{2}$ are rules then Ite $\left(\varphi, r_{1}, r_{2}\right)$ is an if-then-else (ite) rule.

We say that a rule $r$ is well-formed with respect to the type $\iota \rightarrow o$, denoted $r: \iota \rightarrow o$, when one of the following conditions holds:

- $r$ is the rule Undef.

- $r$ is a rule $\operatorname{Base}(f: \iota \rightarrow o)$.

- $r$ is a rule Ite $\left(\varphi: \iota \rightarrow\right.$ bool, $\left.r_{1}: \iota \rightarrow o, r_{2}: \iota \rightarrow o\right)$.

A rule $r: \iota \rightarrow o$ represents a function $\llbracket r \rrbracket$ from $\mathbb{U}_{\iota}$ to $\mathbb{U}_{o} \cup\{\perp\}$ For all $a \in \mathbb{U}_{\iota}$ :

$$
\begin{aligned}
\llbracket U n d e f \rrbracket(a) & \stackrel{\text { def }}{=} \perp \\
\llbracket \operatorname{Base}(f) \rrbracket(a) & \stackrel{\text { def }}{=} \llbracket f \rrbracket(a) \\
\llbracket \operatorname{Ite}\left(\varphi, r_{1}, r_{2}\right) \rrbracket(a) & \stackrel{\text { def }}{=} \begin{cases}\llbracket r_{1} \rrbracket(a), & \text { if } a \in \llbracket \varphi \rrbracket ; \\
\llbracket r_{2} \rrbracket(a), & \text { otherwise. }\end{cases}
\end{aligned}
$$


We now introduce the central definition of a symbolic branching transducer that uses the definition of rules.

Definition 1. A Symbolic Branching Transducer or BST A with input type $\iota$, output type $o$ and state type $\tau$ is a tuple $\left(q^{0}, R, F\right)$, where

- $q^{0} \in \mathbb{U}_{\boldsymbol{\tau}}$ is the initial state;

- $R$ is an input rule of type $(\iota \times \tau) \rightarrow\left(o^{\leq k} \times \tau\right)$, for some $k \geq 0$;

- $F$ is a final rule of type $\tau \rightarrow o^{\leq k}$, for some $k \geq 0$.

For a basic subrule $r=\operatorname{Base}(\lambda(x, y) \cdot\langle f(x, y), g(x, y)\rangle)$ of the input rule, $f$ is called the yield and $g$ the update of $r$. A basic subrule of the final rule is called a final yield.

We write $p \stackrel{a / b}{\longrightarrow} A q$ for a concrete transition of $A$ such that $\llbracket R_{A} \rrbracket(a, p)=(\boldsymbol{b}, q)$. Similarly, we write $q \stackrel{/ b}{\longrightarrow}$ for a final output of $A$ such that $\llbracket F_{A} \rrbracket(q)=\boldsymbol{b}$. Intuitively, a final output is a special case of an input-epsilon move of a classical finite state transducer into a final state, but it is algorithmically useful to keep final rules separate from general input-epsilon moves. Unlike input-epsilon moves in general, final rules do not affect the core algorithms, while providing a very convenient mechanism to yield additional outputs upon reaching the end of the input tape.

We write $A^{\sigma / \gamma ; \tau}$ to indicate the input/output types $\sigma / \gamma$ and the state type $\tau$ of a BSTA. In the following we use the abbreviations $\Sigma=\mathbb{U}_{\iota}, \Gamma=\mathbb{U}_{\boldsymbol{o}}$ and $Q=\mathbb{U}_{\tau}$.

The reachability relation $p \stackrel{\boldsymbol{a} / \boldsymbol{b}}{\longrightarrow}$ A $q$ for $\boldsymbol{a} \in \Sigma^{*}, \boldsymbol{b} \in \Gamma^{*}$, and $p, q \in Q$ is defined through the closure under the following conditions, where '.' is concatenation of sequences:

- For all $q \in Q, q \stackrel{\epsilon / \epsilon}{\longrightarrow}$ A.

- If $p \stackrel{\boldsymbol{a} / \boldsymbol{b}}{\longrightarrow}{ }_{A} p^{\prime} \stackrel{a / c}{\longrightarrow}_{A} q$ then $p \stackrel{\boldsymbol{a} \cdot a / \boldsymbol{b} \cdot \boldsymbol{c}}{\longrightarrow_{A}} q$.

Definition 2. The transduction of a $B S T A, \mathscr{T}_{A}$, is a function from $\Sigma^{*}$ to $\Gamma^{*} \cup\{\perp\}$ :

$$
\mathscr{T}_{A}(\boldsymbol{a}) \stackrel{\text { def }}{=} \begin{cases}\boldsymbol{b} \cdot \boldsymbol{c} & \text { if } \exists q \in Q, \boldsymbol{b}, \boldsymbol{c} \in \Gamma^{*} \text { such that }\left(q_{A}^{0} \stackrel{\boldsymbol{a} / \boldsymbol{b}}{\longrightarrow_{A}} q \stackrel{/ \boldsymbol{c}}{\longrightarrow}_{A}\right) \\ \perp & \text { otherwise }\end{cases}
$$

$B S T$ s are inherently deterministic and single-valued as rules are functions and according to Definition 2 all of the input is consumed before the final rule is applied.

The following example illustrates the use of $B S T$ s on a typical string transformation scenario and illustrates the fragment of $\mathrm{C} \#$ that we use for defining BSTs in this paper.

Example 2.1. The C\# program in Figure 1 corresponds to a $B S T$ that decodes certain occurrences of pairs of digits between 5 and 9 by their corresponding ASCII letters. For example DecodeDigitPairs("a77") is "aM".

Let $f$ be the $\lambda$-term $\lambda(x, y) .((10 *(y-48))+(x-48))$ and let $\varphi$ be the predicate $\lambda(x, y) \cdot\left({ }^{\prime} 5\right.$ ' $\leq$ $x \leq$ ' 9 '). The tree of if else statements in the Update method maps directly to the following input rule where we lift the $\lambda$-prefix to be in the front:

$$
\begin{array}{ll}
\lambda(x, y) . \operatorname{Ite}(y=0, & \operatorname{Ite}(\varphi(x, y), \operatorname{Base}([], x), \operatorname{Base}([x], y)), \\
& \operatorname{Ite}(\varphi(x, y), \operatorname{Base}([f(x, y)], 0), \operatorname{Base}([x], y)))
\end{array}
$$




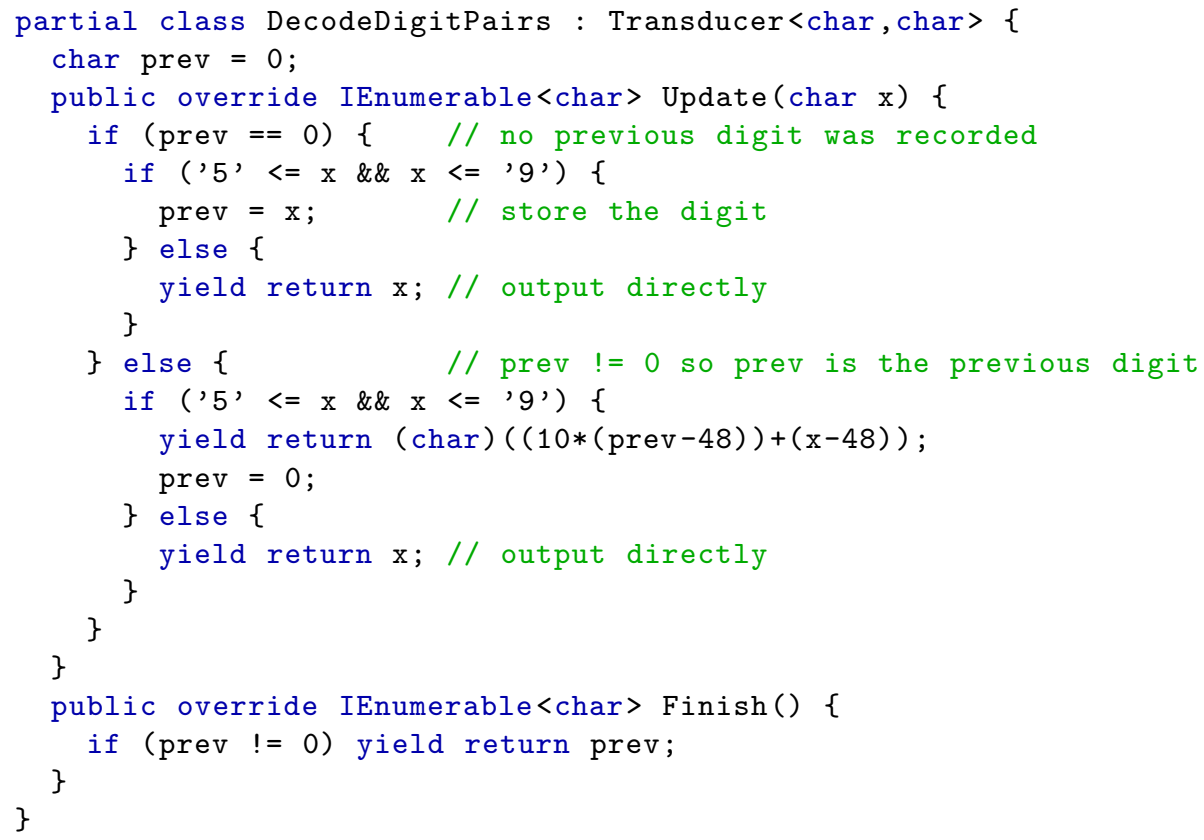

Figure 1: Sample C\# transducer.

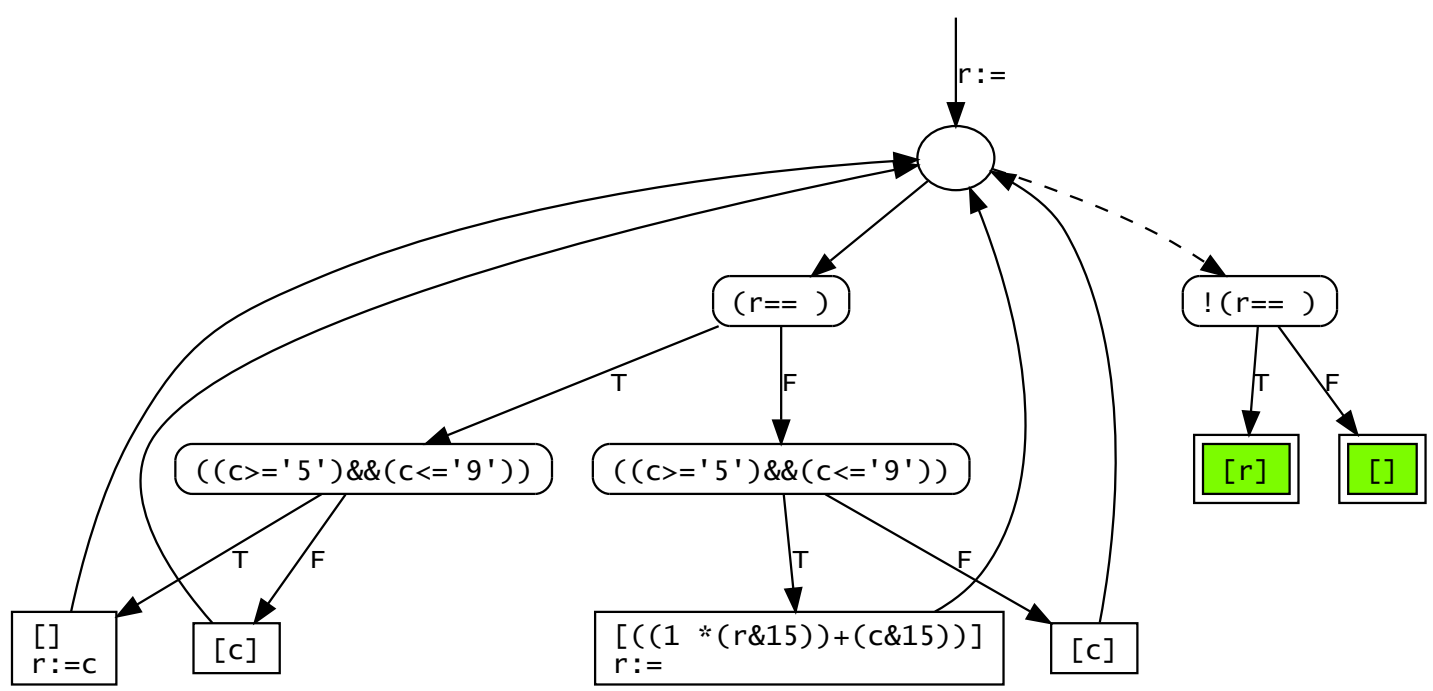

Figure 2: Depiction of the BST in Figure 1. Dashed arrows correspond to final rules. Oval nodes correspond to branch conditions and rectangular nodes correspond to basic rules.

Similarly, the final rule corresponds to the Finish method:

$$
\lambda y . \operatorname{Ite}(y \neq 0,[y],[])
$$




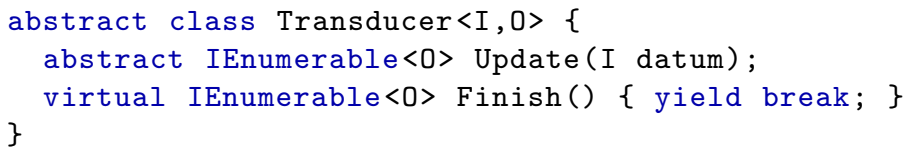

Figure 3: The abstract class for transducers that DecodeDigitPairs Figure 1 extends.

The graphical illustration of the $B S T$ for DecodeDigitPairs is shown in Figure 2. All graphs in the paper are produced automatically from our analysis framework.

\section{C\# to BSTs}

In this section we present a procedure for translating a transducer specified in C\# (see Figure 1), into an equivalent BST (see Figure 2). The C\# code is in the form of a class implementing the Transducer $\langle$ I , 0$\rangle$ interface in Figure 3. The code must:

- produce output via yield return statement,

- not reference variables apart from its parameters, local variables and non-static fields,

- not call functions outside the class or any non-pure functions (purity is checked).

To translate C\# into BSTs the procedure has to be able to lift types and operations on them in $\mathrm{C \#}$ into those in a background logic for a BST. If the background logic is defined by what is supported in Z3 the lifting could for example lift:

- int into 32-bit bitvectors,

- bool into the Boolean type,

- struct into tuples (or algebraic datatypes) of the component types,

The following explanation assumes that an appropriate lifting is available, but does not go into details.

We write a function that maps $a_{1}, \ldots, a_{n}$ to $b_{1}, \ldots, b_{n}$ as $\left\{a_{1} \mapsto b_{1}, \ldots, a_{n} \mapsto b_{n}\right\}$. Given a function $f$ we write the modified function that maps $a$ to $b$ as $f\{a \mapsto b\}$.

The entry point to the procedure is ToBST in Figure 4. Given a program $P$ it constructs control flow graphs (CFGs) [6] for the Update and Finish methods (using GETCFG) and calls $\operatorname{ToRule}_{R}$ (or ToRule $F$ ) to translate the C\# code into rules for a BST. The state type $\tau$ for the final BST is a Cartesian product type of the lifted field types. ToBST also maps (see line 4) the fields of $P$ into an initial variable mapping, where each field is mapped to a term that projects the appropriate value out of the state. This initial mapping represents an identity transformation on the state. To construct the initial state $q_{0}$, ToBST lifts the initial values of the fields of $P$ into the background logic and constructs the appropriate product from them. Finally, ToBST returns a BST with $q_{0}$, where the input and final rules implement the Update and Finish methods respectively.

The main procedure for translating C\# into input rules is $\operatorname{ToRuLE}_{R}$ in Figure 5. In addition to a basic block $B$ from a CFG and the current variable assignment vars, each call to ToRULE ${ }_{R}$ is passed a path constraint $\varphi_{\text {path }}$. As ToRulE $R$ recursively calls itself to explore further basic blocks, the recursion structure will correspond to the tree of possible executions of the current 


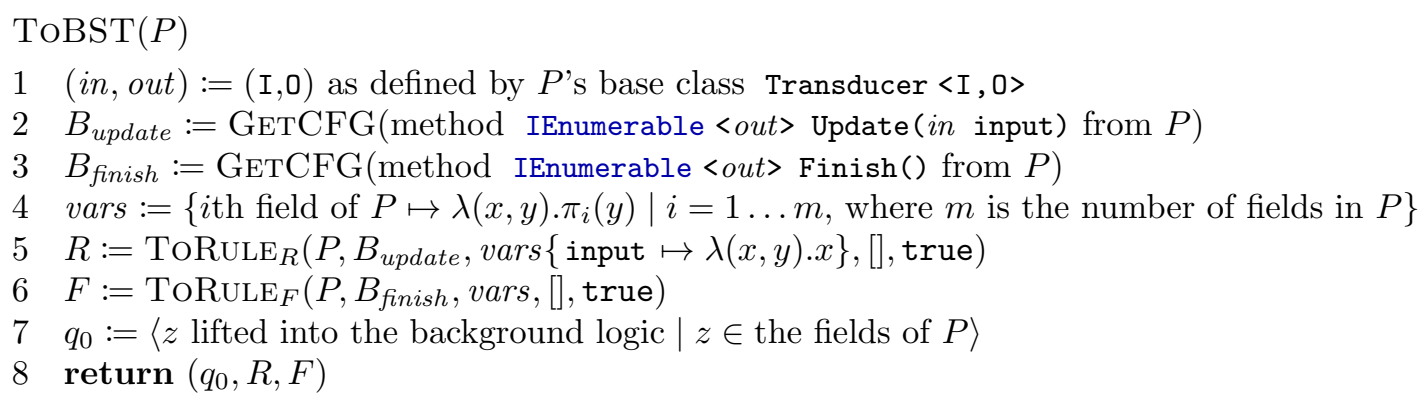

Figure 4: Translation of C\# into a BST

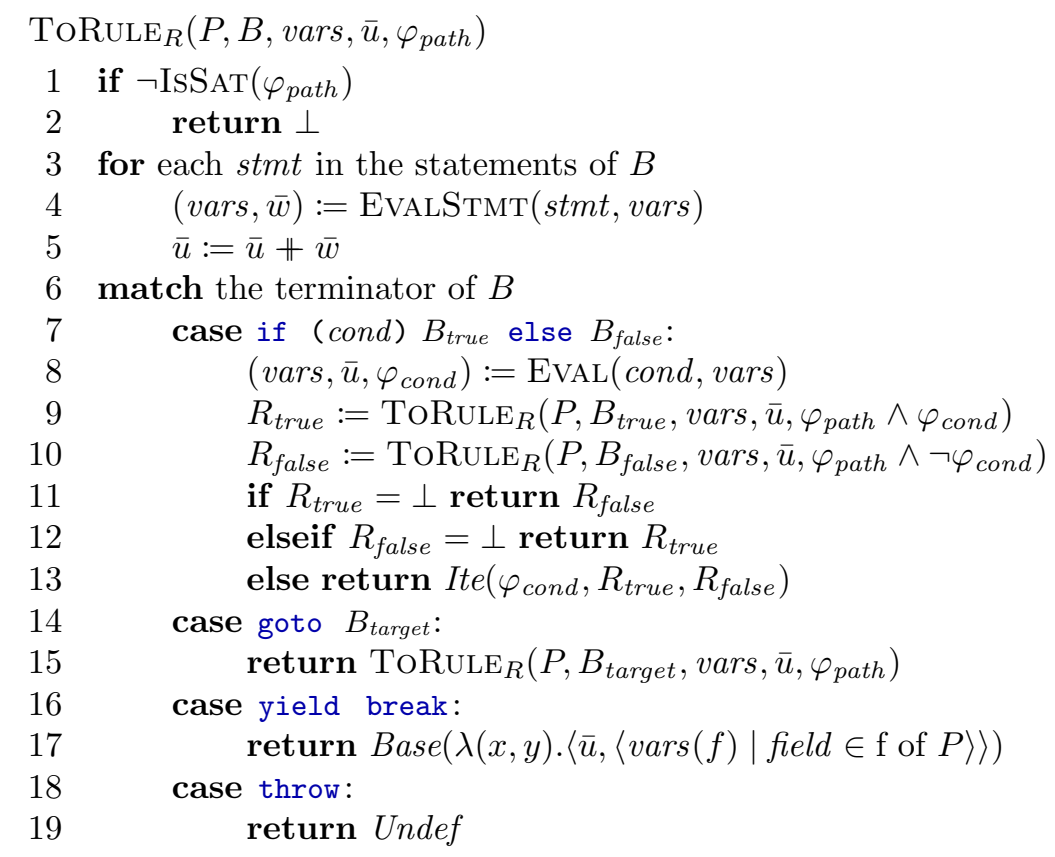

Figure 5: Translation of a CFG into a rule

CFG. In each recursive call $\varphi_{\text {path }}$ is the conjunction of branch constraints for the corresponding execution path. On line 1 satisfiability of $\varphi_{\text {path }}$ is checked using an SMT solver to prune paths from the rule being constructed.

For obtaining final rules ToRule $F$, which is not shown, is used. The only difference to $\operatorname{ToRuLE}_{R}$ is that on line 17 the returned base rule does not specify a state update.

The basic block $B$ consists of a list of non-branching statements followed by a terminator. $\operatorname{ToRule}_{R}$ executes the statements in the basic block by calling EvalStmt, which returns an updated variable assignment and a list of yields. The code on lines 6-19 that pattern matches on the terminator of $B$ handles the different types of control flow: 
if else causes the exploration to branch into two recursive ToRULE $R$ calls. The path constraint of the recursive calls to $\mathrm{ToRuLE}_{R}$ may end up being unsatisfiable, in which case the rule simplifies to the one from the other branch instead of an Ite-rule.

goto has one target and as such the recursive call is a tail call, i.e., for efficiency this call could just set the parameters in the current call and jump to the beginning of the procedure.

yield break terminates the current execution path. A Base-rule is constructed from the list of yields along the path and the state update as defined by the values in vars for the fields of $P$.

throw results in an Undef-rule, indicating that the input was rejected.

This process of exploring an execution tree of the CFG while pruning unsatisfiable branches also supports looping constructs in C\#, since these translate to a CFG with if else and goto terminators. As long as the loops terminate for all inputs and states (also unreachable ones), ToRule $_{R}$ will also terminate. However, it is easy to use loops to specify very large rules, in which case $\operatorname{ToRulE}_{R}$ may run out of memory or appear to hang.

Example 3.1. The following transducer formats unsigned 32-bit integers in decimal notation.

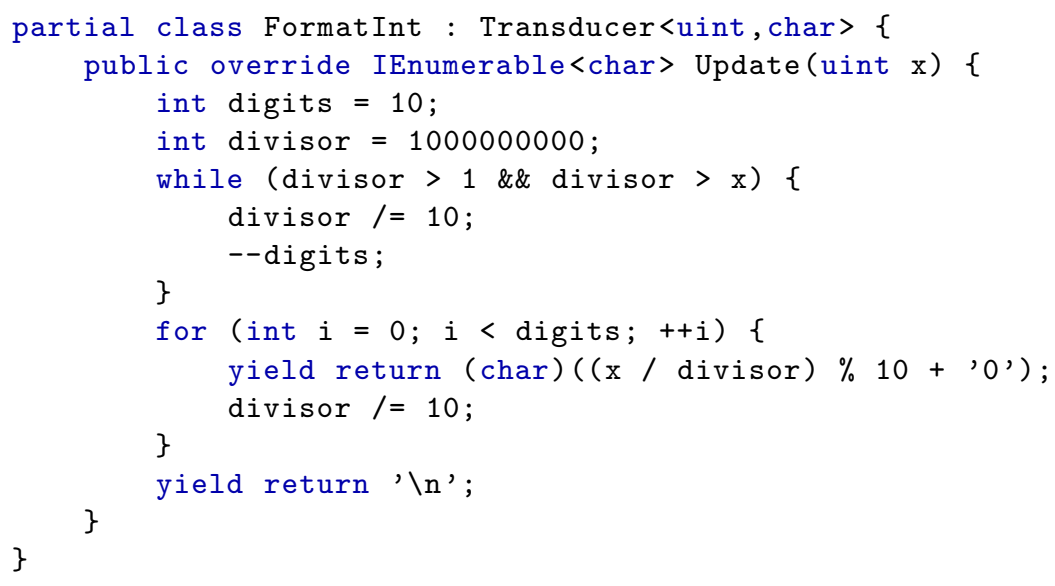

The input rule for this transducer has ten different Base-rules (one for each number of digits). A transducer written in C\# without loops would be larger and the code would have more repetition.

The procedure for translating C\# statements is EvalStmT in Figure 6, which directly handles:

- yield return statements by returning the expression evaluated with EvAL in the list of yields, and

- local variable definitions by returning an updated vars.

For statements which consist of just a C\# expressions it calls EvAL, which interprets the expression in the context of the current vars and returns an equivalent expression in the background logic. Since expressions may have side effects, EvAL also returns an updated version of vars.

The handling of function calls on lines 6-13 of Figure 6 calls for further explanation. To evaluate the function $f$ its CFG is created and interpreted by a call to the procedure ToExPR 

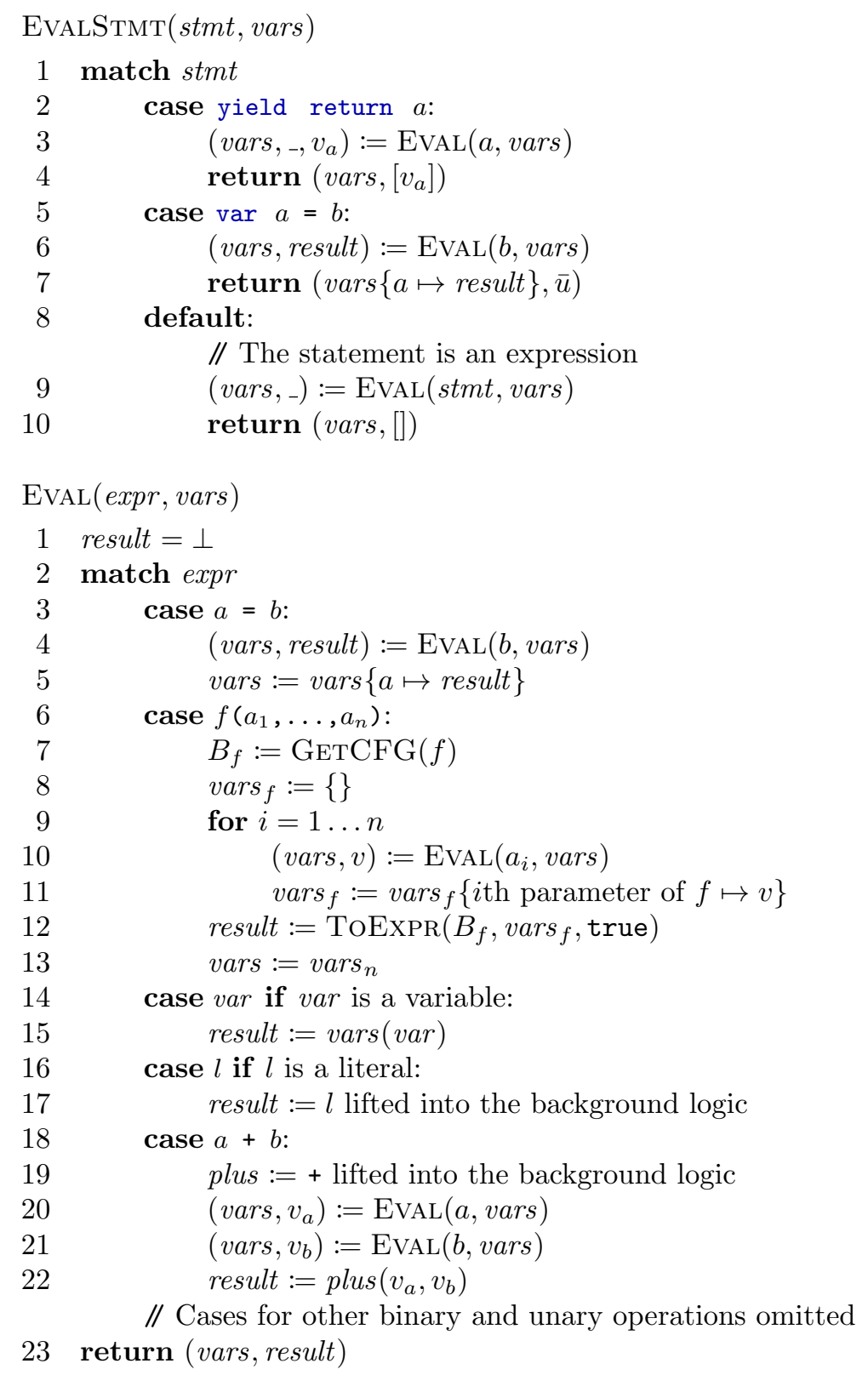

Figure 6: Translation of C\# expressions into a background logic

in Figure 7. The arguments are interpreted left-to-right (applying any side effects in vars) and an initial variable assignment vars $f$ mapping the parameters of $f$ to the arguments is created.

ToExpR is largely similar to TORULE $_{R}$ in Figure 5, except that it constructs a formula in the background logic instead of rules. The main differences are in the terminators supported, 


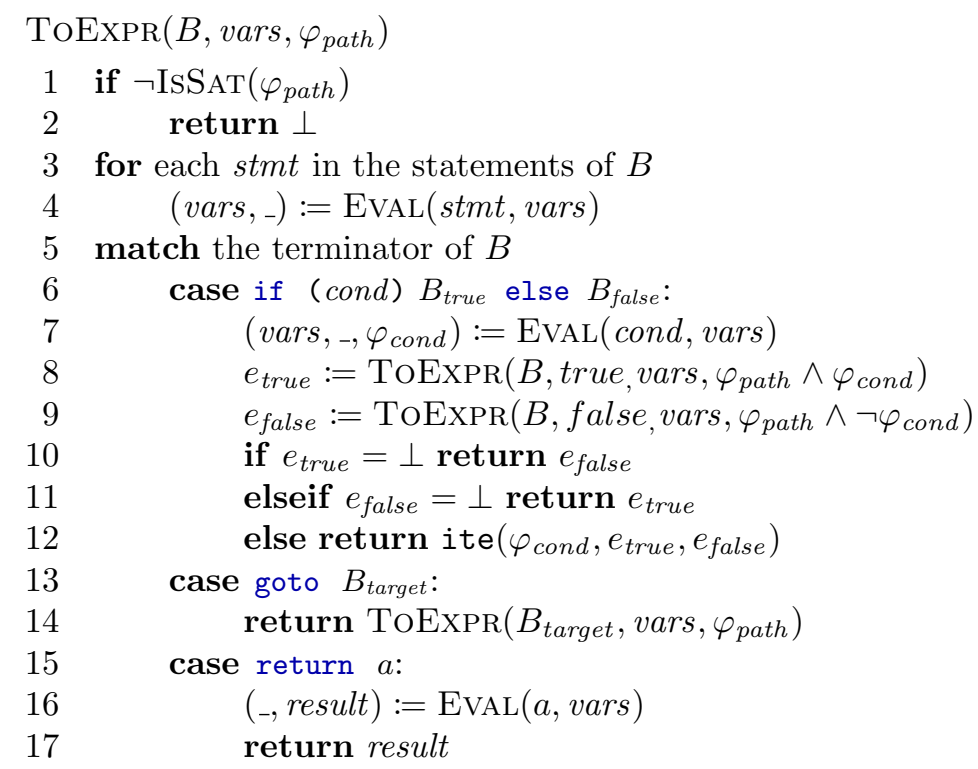

Figure 7: Translation of a CFG for a pure function into an expression in a background logic

with functions passed to ToExPR having to end in a return statement and yield statements not being supported. Also note that on line 12 the ite being returned is a term in the background logic instead of an Ite-rule. As the initial vars $f$ constructed by EvaL includes only the parameters of $f$, only pure functions are supported by ToExPR.

While the Eval presented here uses ToExpr to inline function calls, ToExpR can also be used to provide background definitions for functions. This would result in more compact terms being created, but would prevent functions from being simplified to the context they are called from.

\section{Register to Control State Exploration}

In this section we develop an algorithm that allows us to eliminate either all or some of the state registers used in a deterministic $B S T A$. In particular, we focus on two, most prominent cases:

- full exploration, and

- Boolean exploration.

For the purpose of explaining the exploration algorithm, we extend $A=\left(q^{0}, R, F\right)$ with a component $P$ that is a finite set of control states and an initial control state $p^{0} \in P$. The rules $R$ and $F$ are extended to be maps from $P$ to rules, and each basic subrule of the input rule $R$ has an additional control state component $p \in P$. With this extension in mind, we write a basic rule as Base (yield, update, $p$ ). We say that $A$ is stateless when the register type $\tau$ is the unit type T0 $\left(\mathbb{U}_{\mathbf{T} 0}=\{\langle\rangle\}\right)$, i.e., registers are not used in a stateless $B S T$, and thus $R$ has the 

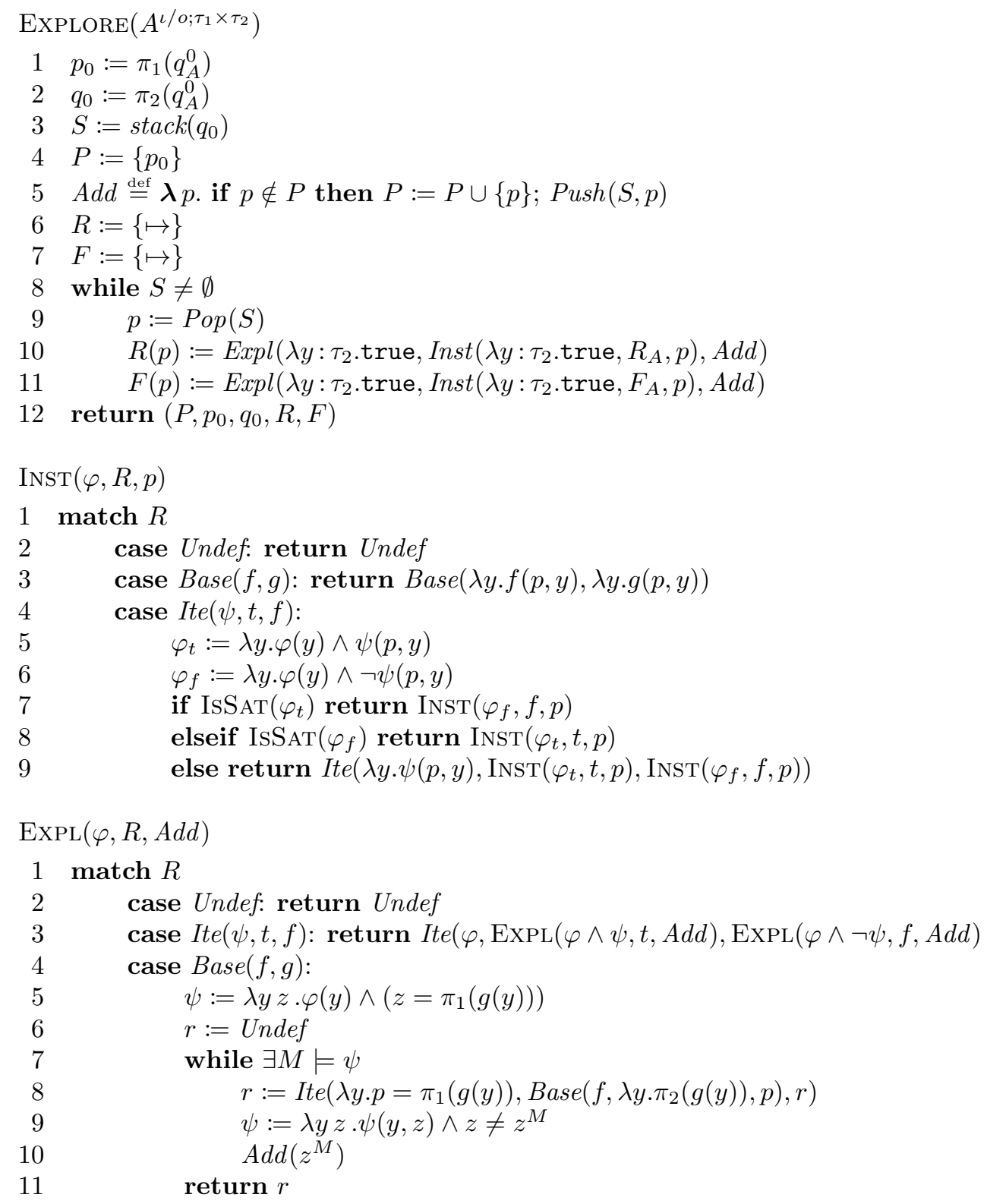

Figure 8: Exploration algorithm of $B S T \mathrm{~s}$.

equivalent form

$$
\left\{p_{1} \mapsto r_{1}, p_{2} \mapsto r_{2}, \ldots, p_{|R|} \mapsto r_{|R|}\right\}
$$

where each rule $r_{i}$ corresponds to a conditional statement that may yield outputs and transition to new control states but does not make use of registers by storing intermediate results in registers. This extension is useful for separation of concerns, it helps to keep the control state 


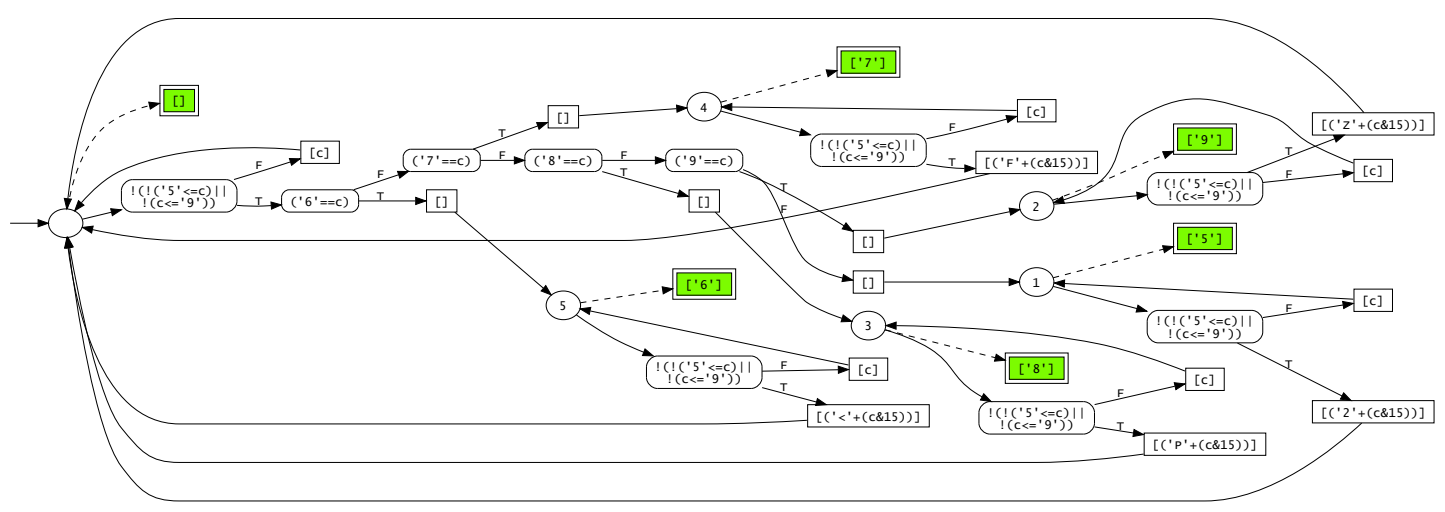

Figure 9: BST after full exploration of DecodeDigitPairs in Figure 1.

separate from the data state. For example, the $B S T$ is Example 2.1 is not stateless because the rules depend on the register $y$.

By full exploration of $A$, we mean a construction of a stateless $B S T A^{\mathrm{f}}$ such that $\mathscr{T}_{A}=\mathscr{T}_{A^{\mathrm{f}}}$, i.e., $A$ and $A^{\mathrm{f}}$ are equivalent. Full exploration is not always possible, because equivalence of stateless BSTs reduces to equivalence of symbolic finite transducers (SFTs), and equivalence of SFTs is decidable [16] modulo a decidable label theory, while equivalence of BSTs is undecidable already for very restricted decidable label theories. Even when full exploration is possible, $A^{\mathbf{f}}$ may still be exponentially larger than $A$.

By Boolean exploration of $A$, we mean a construction of an $B S T A^{\mathbf{b}}$ such that $\mathscr{T}_{A}=\mathscr{T}_{A^{\mathrm{b}}}$ where all Boolean registers of $A$ have been eliminated. For example, if the state type of $A$ is (bool $\times$ bool) $\times$ int then the the state type of $A^{\mathbf{b}}$ is int, i.e., the two Boolean registers have been eliminated by adding new control states.

Note that, in order to completely eliminate the symbolic update of a rule $\operatorname{Base}([], \lambda(x, y) \cdot \varphi(x))$, where $\varphi$ is a $\iota$-predicate, i.e., to replace $\varphi$ by $\lambda$ x.true (resp. $\lambda x$.false) we would need to decide if $\forall x \varphi(x)$ holds, i.e., $\neg \varphi$ is unsatisfiable, (resp. if $\forall x \neg \varphi(x)$ holds, i.e., $\varphi$ is unsatisfiable).

Algorithm. The generic exploration algorithm of BSTs is described in figure 8. The algorithm takes as its input a $B S T A$, and assumes a projection of the state type $\tau$ of $A$ into two parts $\tau_{1}$ and $\tau_{2}$. We assume, without loss of generality, that $\tau=\tau_{1} \times \tau_{2}$. The algorithm uses an SMT solver to solve satisfiability and to generate models for formulas.

The algorithm generates a new $B S T$ by exploring the rules with respect to $\tau_{1}$, effectively eliminating $\tau_{1}$, i.e. turning it into an explicit state. In order to avoid special cases, we may always assume that either $\tau_{1}$ or $\tau_{2}$ can be unit types $\mathrm{T} 0\left(\mathbb{U}_{\mathbf{T} 0}=\{\langle\rangle\}\right)$. Now, full exploration of $A$ corresponds to the case when $\tau_{2}$ is unit type, and Boolean exploration corresponds to the case when $\tau_{1}$ is a Cartesian combination of Boolean registers and $\tau_{2}$ is a Cartesian combination of all the non Boolean registers.

$\operatorname{INST}(\varphi, r, p)$ creates an instance of the rule $r$ with the path condition $\varphi$ with respect to the fixed register values given by $p$. For the exception rule this is a no-op. For a basic rule this is a partial instantiation of the yield and update with respect to $p$, where $\lambda y \cdot f(p, y)$ instantiates the first projection of the state register with the value $p$. An important point for the rules is that unreachable rule instances are incrementally eliminated by deciding satisfiability of corresponding accumulated path conditions.

$\operatorname{ExpL}(\varphi, r, a d d)$ is a form of partial exploration of $r$ the with respect to $\tau_{1}$ or the projection projection function. For the exception rule the operation is a no-op. For an if-then-else rule, the 
step is a direct propagation of the concretizations of the branches. The core of the computation takes place during the concretization of basic rules.

Theorem 4.1. Let $A$ be a deterministic $B S T$ with state type $\tau_{1} \times \tau_{2}$. If $\operatorname{Explore}(A)$ terminates then the result is a $B S T$ that is equivalent to $A$ and whose state type is $\tau_{2}$.

We omit the formal proof of the theorem but note that termination of the algorithm depends on two factors: decidability of the background theory, and finiteness of the reachable subset of $\mathbb{U}_{\tau_{1}}$. The first point is already needed in the INST procedure that eliminates unsatisfiable branches. The second point is needed both, for termination of construction of $r$ in ExPL, as well as for guaranteeing that the search stack is bounded in size. A sufficient condition for the second point is when the functions used for computing the first state projection have the finite-range property, i.e., when $\mathbb{U}_{\tau_{1}}$ can be assumed to be finite.

Example 4.1. The $B S T$ after full exploration of DecodeDigitPairs from Figure 1, is illustrated in Figure 9. The unexplored $B S T$ (in Figure 2) has a single control state 0, while the fully explored $B S T$ has 6 control states.

\section{Implementation}

We have implemented the C\# to BSTs and the register exploration algorithms in the Automata library, which is available under the MIT license. A version with our changes can be found at:

https://github.com/0lliSaarikivi/Automata/

The C\# frontend is implemented in the CSharpFrontend project. A string containing a class extending Transducer $<\mathrm{I}, 0>$ can be turned into an instance of $\mathrm{STb}<\mathrm{FuncDecl}$, Expr, Sort $>$ (a BST with Z3 formulas as its background logic) by calling:

Microsoft. Automata.CSharpFrontend.CSharpParser.FromString (Z3Context, string)

For Boolean exploration STb has an ExploreBools() method.

\section{Related Work}

Symbolic transducers were introduced in flat form in [16] for analysis of string sanitizers with the main focus on symbolic finite transducers or SFTs. The paper [14] develops composition algorithms for BSTs. Further work on symbolic transducers has focused on register exploration and input grouping. Input grouping tries to take advantage of grouping characters into larger tokens in order to avoid intermediate register usage, that has applications in decoder analysis [7] and parallelization [17].

Stream processing area has a large body of work [9, 10, 11, 12, 15]. Some libraries for streams provide APIs for expressing stateful operations. The Apache Flink [5] and Spark Streaming [4] distributed streaming engines both provide support for using state in stream operations and an associated framework for implementing fault tolerance in the presence of state. The Highland.js [3] and Conduit [2] are traditional stream libraries, which both provide a way to express stateful operations. 


\section{Conclusion}

The translation of C\# into BSTs in Section 3 allows a natural and compact way to specify effectful comprehensions as imperative code. Using a fragment of the host language for specification ensures a seamless integration by obviating impedance mismatches arising from differences in type systems.

The register exploration algorithm in Section 4 exposes control states in the BST, thus allowing the programmer to freely use C\#'s native types for state while still permitting efficient application of BST algorithms that leverage control state, such as fusion [14].

\section{References}

[1] Apache Hadoop. http://hadoop.apache.org/.

[2] Conduit (Haskell library). https://github.com/snoyberg/conduit.

[3] Highland.js. http://highlandjs.org/.

[4] Spark Streaming. http://spark.apache.org/streaming/.

[5] Alexander Alexandrov, Rico Bergmann, Stephan Ewen, Johann-Christoph Freytag, Fabian Hueske, Arvid Heise, Odej Kao, Marcus Leich, Ulf Leser, Volker Markl, Felix Naumann, Mathias Peters, Astrid Rheinländer, Matthias J. Sax, Sebastian Schelter, Mareike Höger, Kostas Tzoumas, and Daniel Warneke. The Stratosphere platform for big data analytics. The VLDB Journal, 23(6):939964, December 2014.

[6] Frances E. Allen. Control flow analysis. SIGPLAN Not., 5(7):1-19, July 1970.

[7] Loris D'antoni and Margus Veanes. Extended symbolic finite automata and transducers. Formal Methods in System Design, 47(1):93-119, August 2015.

[8] Jeffrey Dean and Sanjay Ghemawat. MapReduce: Simplified data processing on large clusters. Commun. ACM, 51(1):107-113, January 2008.

[9] Denis Debarbieux, Olivier Gauwin, Joachim Niehren, Tom Sebastian, and Mohamed Zergaoui. Early nested word automata for XPath query answering on XML streams. Theoretical Computer Science, 578:100-125, May 2015.

[10] Andreas Maletti, Jonathan Graehl, Mark Hopkins, and Kevin Knight. The power of extended top-down tree transducers. SIAM J. Comput., 39(2):410-430, June 2009.

[11] Tova Milo, Dan Suciu, and Victor Vianu. Typechecking for XML transformers. In Proceedings of the Nineteenth ACM SIGMOD-SIGACT-SIGART Symposium on Principles of Database Systems (PODS'00), pages 11-22. ACM, 2000.

[12] Barzan Mozafari, Kai Zeng, Loris D'antoni, and Carlo Zaniolo. High-performance complex event processing over hierarchical data. ACM Trans. Database Syst., 38(4):21:1-21:39, December 2013.

[13] Todd A. Proebsting and Scott A. Watterson. Filter fusion. In Proceedings of the 23rd ACM SIGPLAN-SIGACT Symposium on Principles of Programming Languages (POPL'96), pages 119130. ACM, 1996.

[14] Olli Saarikivi, Margus Veanes, Todd Mytkowicz, and Madan Musuvathi. Fusing effectful comprehensions. In Proceedings of the 38th ACM SIGPLAN Conference on Programming Language Design and Implementation (PLDI'17). ACM, 2017.

[15] Jesper H. Spring, Jean Privat, Rachid Guerraoui, and Jan Vitek. StreamFlex: High-throughput stream programming in Java. In Proceedings of the 22nd Annual ACM SIGPLAN Conference on Object-Oriented Programming Systems and Applications (OOPSLA'07), pages 211-228. ACM, 2007.

[16] Margus Veanes, Pieter Hooimeijer, Benjamin Livshits, David Molnar, and Nikolaj Bjorner. Symbolic finite state transducers: Algorithms and applications. In Proceedings of the 39th Annual ACM 
SIGPLAN-SIGACT Symposium on Principles of Programming Languages (POPL'12), pages 137150. ACM, 2012.

[17] Margus Veanes, Todd Mytkowicz, David Molnar, and Benjamin Livshits. Data-parallel stringmanipulating programs. In Proceedings of the 42nd Annual ACM SIGPLAN-SIGACT Symposium on Principles of Programming Languages (POPL'15), pages 139-152. ACM, 2015.

[18] Philip Wadler. Deforestation: Transforming programs to eliminate trees. Theoretical Computer Science, 73(2):231-248, January 1988.

[19] Matei Zaharia, Mosharaf Chowdhury, Michael J. Franklin, Scott Shenker, and Ion Stoica. Spark: Cluster computing with working sets. In Proceedings of the 2nd USENIX Conference on Hot Topics in Cloud Computing (HotCloud'10), pages 10-10. USENIX Association, 2010. 